Review Article

\title{
Human Cytomegalovirus Latency: Targeting Differences in the Latently Infected Cell with a View to Clearing Latent Infection
}

\author{
Emma Poole, Mark Wills, and John Sinclair \\ Department of Medicine, Cambridge University, Addenbrooke's Hospital, Hills Road, Level 5, P.O. Box 157, \\ Cambridge CB2 0QQ, UK \\ Correspondence should be addressed to John Sinclair; js@mole.bio.cam.ac.uk
}

Received 16 January 2014; Accepted 24 March 2014; Published 4 June 2014

Academic Editor: Qiyi Tang

Copyright (C) 2014 Emma Poole et al. This is an open access article distributed under the Creative Commons Attribution License, which permits unrestricted use, distribution, and reproduction in any medium, provided the original work is properly cited.

\begin{abstract}
Human cytomegalovirus (HCMV) is a human herpesvirus which causes little or no disease in the immunocompetent. However, in immunocompromised individuals, neonates, or patients on immune suppressive therapies, HCMV can cause significant morbidity and mortality in some patient groups. As with all herpesviruses, HCMV has two life cycle phases: a productive phase, where new virions are produced and a latent phase where there is a restricted gene transcription profile and no new virion production. Currently available antivirals target the productive phase of HCMV infection and, although these have greatly decreased the severity of HCMV-induced disease in immunocompromised or immunosuppressed individuals, they often have associated toxicities, routinely result in selection of drug resistant viral mutants, and, importantly, they do not target cells latently infected with virus. Thus, there is a real need to derive novel antiviral therapies which, not least, are also able to target latent infection. In this paper, we describe recent work which has begun to analyse changes in the cell associated with latent infection and the possibility that these latencyassociated changes in cell phenotype could be targeted by novel chemo- or immunotherapeutic strategies in order to diminish, or even clear, latent infection at least in some specific clinical settings.
\end{abstract}

\section{Review of the Literature and the Development of Tissue Culture Latency Models}

1.1. Epidemiology and Clinical Aspects. Human cytomegalovirus (HCMV) is a pathogen that can cause significant morbidity and mortality, particularly in the immunocompromised host [1-10]. For instance, HCMV disease is regularly seen after infection of individuals with a suppressed immune system, such as transplant patients or those suffering from AIDS. In such individuals, HCMV-associated pathology is observed in a range of tissues and can lead to an array of diseases including retinitis and pneumonitis as well as bowel and heart disease $([1,3,4,10]$ and see Figure 1). Similarly, infection in utero, in the developing immunonaive foetus, can result in mental retardation, deafness, and blindness [59] which impact substantially QUALYS (quality adjusted life years) measurements and, for these reasons, HCMV has been named as a high vaccine priority [11].

In the immunocompetent host, however, primary HCMV infection rarely causes disease and this is likely due to the well-established robust immune response to the virus. Indeed, in the developed world, $50-90 \%$ of populations can be HCMV seropositive (and this can be as high as $100 \%$ in some populations in the developing world), yet HCMV disease is rarely a problem in otherwise healthy seropositive carriers.

Despite this rigorous host immune control, HCMV is never cleared after primary infection but persists for the lifetime of the host which is facilitated by the ability of the virus to establish a latent infection during which time virus is carried silently in certain cell types in the absence of new virion production [12]. A consensus view is that, in healthy carriers, latent virus is able to periodically reactivate 


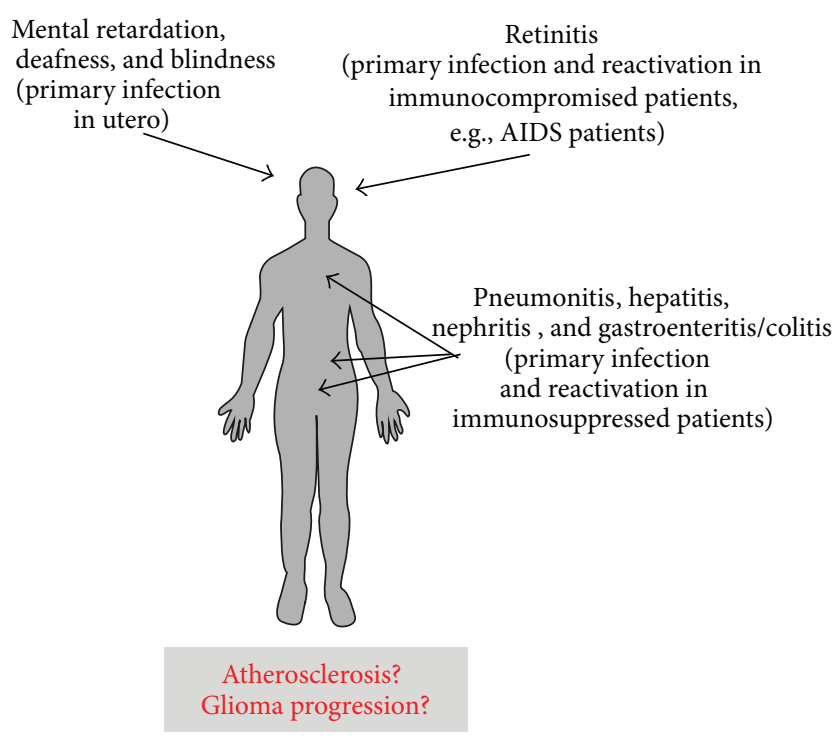

Figure 1: HCMV-induced disease pathology. HCMV causes disease in individuals where the immune system is compromised. Mental retardation, deafness, and blindness can occur following in utero primary infection [5-8]. Retinitis occurs following primary infection and reactivation in AIDS patients [23]. Pneumonitis, hepatitis, nephritis, and gastroenteritis/colitis can occur following immune suppression $[1,4,10,18,24,25]$. HCMV has also been suggested to play a role in atherosclerosis and glioma progression $[13,16,17,20$, $21,26-28]$.

but, in these immunocompetent individuals, these reactivation events are also subclinical. However, it has also been suggested that this may not be totally unproblematic as there is increasing evidence associating HCMV persistence with long-term diseases (i.e., atherosclerosis, chronic graft rejection, and, perhaps, neoplasias) and reactivation of latent HCMV is likely to be a major source of virus contributing to its long-term carriage [13-22].

In some clinical settings, however, virus reactivation can add substantially to disease burden. For instance, in both solid organ and stem cell transplantation scenarios, when recipients are heavily immunosuppressed, reactivation of the transplant recipient's own virus or, indeed, reactivation of virus from donor material can lead to life threatening disease $[29,30]$.

Current antiviral therapies have clearly resulted in dramatically improved outcomes in, for instance, transplant patients. However, the usefulness of these drugs is often limited by poor bioavailability, associated toxicities, and the development of virus resistance. Similarly, so far, an effective vaccine for the prevention of HCMV infection remains elusive, although some success has been obtained with posttransplant vaccination based on attenuated virus, viral subunits, or plasmid DNA which have been shown to reduce the duration of viraemia and requirement for antiviral treatment in some transplant settings [31-33]. Thus, a need for more effective vaccines or better drug treatments for $\mathrm{HCMV}$-associated disease, particularly after reactivation, is of paramount importance. For these reasons, unraveling the mechanisms by which HCMV maintains life-long persistence and translating this knowledge into novel strategies to target latent infection therapeutically are of real importance.

1.2. General Molecular Biology. HCMV is the prototypic member of the betaherpesvirus subfamily with a large genome of around $230 \mathrm{~kb}$. This is known to encode an array of genes which interdict in numerous normal cellular functions to ensure efficient infection and life-long persistence resulting in a complex life cycle with multiple interactions between the virus and its host.

Following primary infection with HCMV, two outcomes can ensue. The virus can enter the lytic phase of replication in which the viral transcription programme is extensive across the whole of the genome and comprises a temporal cascade of expression of the viral major immediate early genes followed by early then late gene expression. This programme of lytic transcription leads to release of infectious virions and can occur in an array of cells and tissues in vivo [29, 42]. Alternatively, in certain cell types, the virus can enter a latent life cycle which is associated with a much more limited viral transcription programme and a lack of virion production $[37,40,43,44]$. Whilst much is known about the lytic cycle of HCMV, the regulation of viral gene expression, as well as virus/host interactions, during latency is less well understood. In this review, we focus on such analyses which are the main programme of our own research as well as a number of other laboratories, internationally.

1.3. Latent Carriage of HCMV Occurs in Cells of the Myeloid Lineage and Virus Can Be Reactivated upon Differentiation. It is now well established that one site of HCMV latency in vivo is in cells of the myeloid lineage (Figure 2), including CD14+ monocytes and their CD34+ progenitor [29, 37, 40, 44, 49]. In these cells, likely as a concerted effect of a lack of viral activators [39], the presence of latency-associated repressors [40], and the dominance of cellular transcriptional repressors of the MIEP [34, 35, 38, 50], the chromatin around the viral MIEP becomes heavily repressive which suppresses lytic transcription (Figure 2) and maintains latent infection [36, 37]. Following differentiation into macrophages or dendritic cells (DCs), however, changes in the nuclear environment result in chromatin-mediated activation of the viral MIEP and reactivation of lytic replication $[36,37]$. In the context of an inadequate immune response (in either transplant patients or the immunocompromised or the immunonaive), these reactivation events then result in virus dissemination to multiple target organs and subsequent clinical disease.

Latent viral genomes are clearly present in CD34+ haematopoietic progenitors, yet they appear to be carried selectively down the myeloid lineage as they are not detectable in T or B cells ([48] and see Figure 3). Whilst the myeloid lineage is clearly one site of true latency of HCMV in healthy carriers, it is yet to be determined if there are other sites of latency in vivo. For instance, endothelial progenitor cells have been suggested to be a potential additional site of latency [51]. However, latent genomes are not detectable in endothelial cells of the microvasculature which rules out these cells as latent reservoirs [52], but it is difficult ethically 


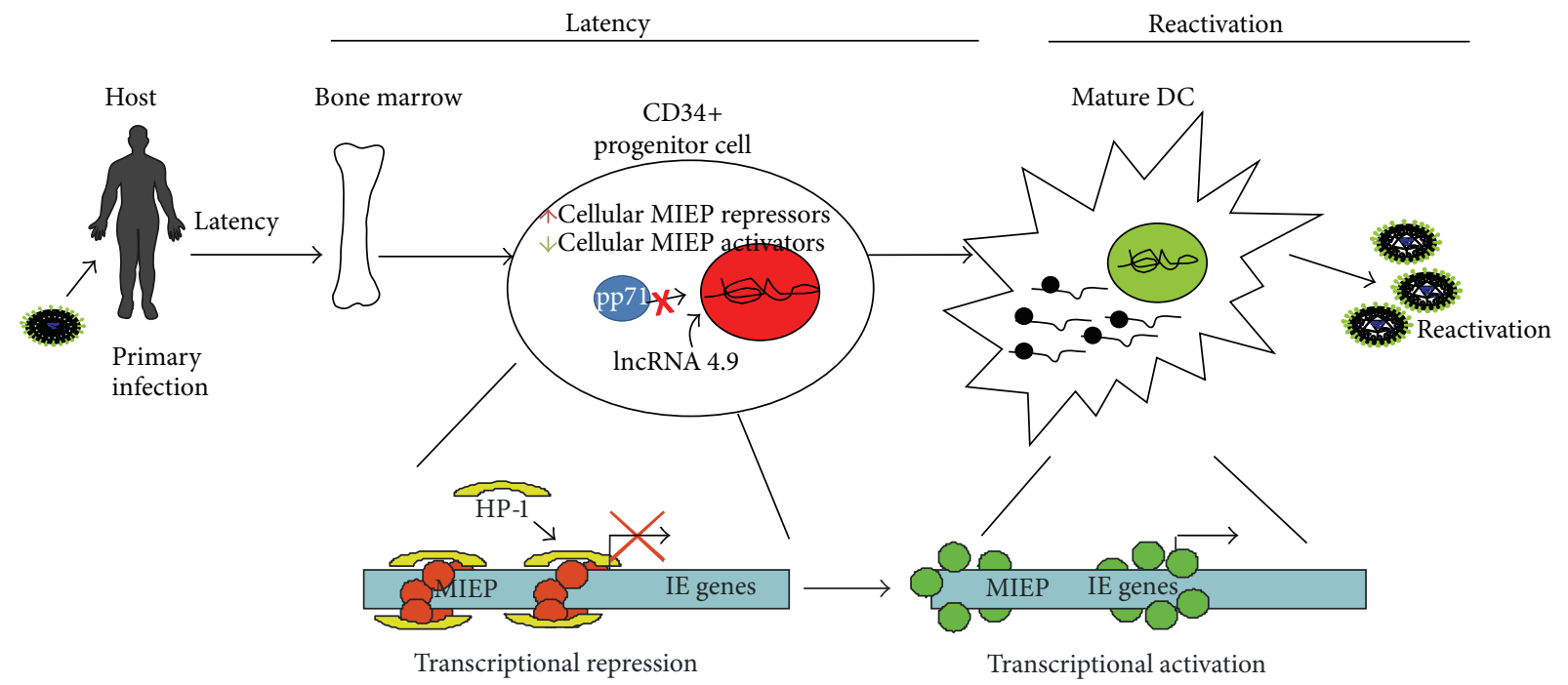

FIGURE 2: HCMV latency and reactivation. Following primary infection, latency is established in the myeloid progenitor CD34+ cells which reside in the bone marrow. Viral genome is associated with chromatin markers of repression in these cells. In particular the major immediate early promoter (MIEP) is associated with a high number of cellular transcriptional repressors and a low number of cellular transcriptional activators [2-4,34-38]. This is likely to be facilitated by an absence of the viral activator pp71 [39] concomitant with the presence of long noncoding RNA (lnr) 4.9 which is thought to interact with the polycomb repressor complex to inhibit transcription [40]. Following differentiation along the myeloid lineage of CD34+ cells into dendritic cells (DCs), the chromatin structure at the MIEP becomes associated with cellular activators of transcription and immediate early gene expression is induced and reactivation of the virus occurs [36, 37, 41].

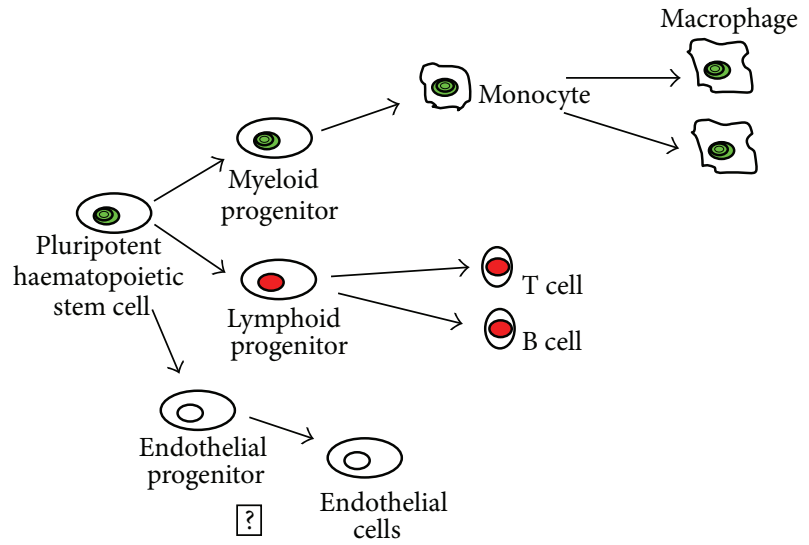

FIGURE 3: HCMV latency is established in myeloid progenitors and reactivation occurs following differentiation. HCMV latency can be established in pluripotent haematopoietic cells which can be differentiated upon specific stimuli into different cell types as shown. HCMV is primarily carried along the myeloid lineage [41, 46, 47] and not the lymphoid lineage [48]. However, whether HCMV is carried along the endothelial cell lineage is uncertain.

to interrogate endothelial cells of, for example, the macrovasculature from otherwise healthy donors to determine if these cells may be additional sites of latency.

Regardless, the mechanisms which regulate the establishment and maintenance of HCMV latency in, and reactivation from, cells of the myeloid lineage are still poorly understood yet are crucial for a full understanding of this persistent human pathogen.
1.4. Experimental Models of HCMV Latency in Cell Lines and Primary Cells. Although naturally latent myeloid cells have been used for some analyses of HCMV latent infections $[36,40,48,49,53,54]$, the number of cells which carry naturally latent viral genome is known to be extremely low (between 1 in 10,000 and 1 in 100,000 [53]). Therefore, it has been necessary to develop experimental models of latency which depend on experimental infection and establishment of latency in long-term cultures which lend themselves more easily to analysis. In our laboratory, and others, a number of models of experimental latency have been established for in vitro analysis of HCMV latency and reactivation. These include primary myeloid progenitors such as granulocyte macrophage progenitors (GMPs) or CD34+ haematopoietic progenitor cells as well as CD14+ monocytes [37, 49, 55-59]; all can be cultured and experimentally infected to establish a latent infection which can be reactivated by differentiation signals-which very much reflects models of natural latency. Figure 4 shows an example of an experimental latency model using primary CD34+ cells. Using this model, it has been demonstrated that, following the establishment of latency, the viral major immediate early promoter enhancer (MIEP) (which drives initial expression of the major immediate early genes (major IEs) required for lytic infection) is associated with repressive chromatin markers. These include methylated histone marks as well as the presence of repressor proteins such as heterochromatin protein 1 (HP1). However, following differentiation of these cells to Langerhans-like dendritic cells, the MIEP becomes associated with markers of active chromatin, such as acetylated H4. Consistent with this, expression of IE72, which is the viral major immediate 


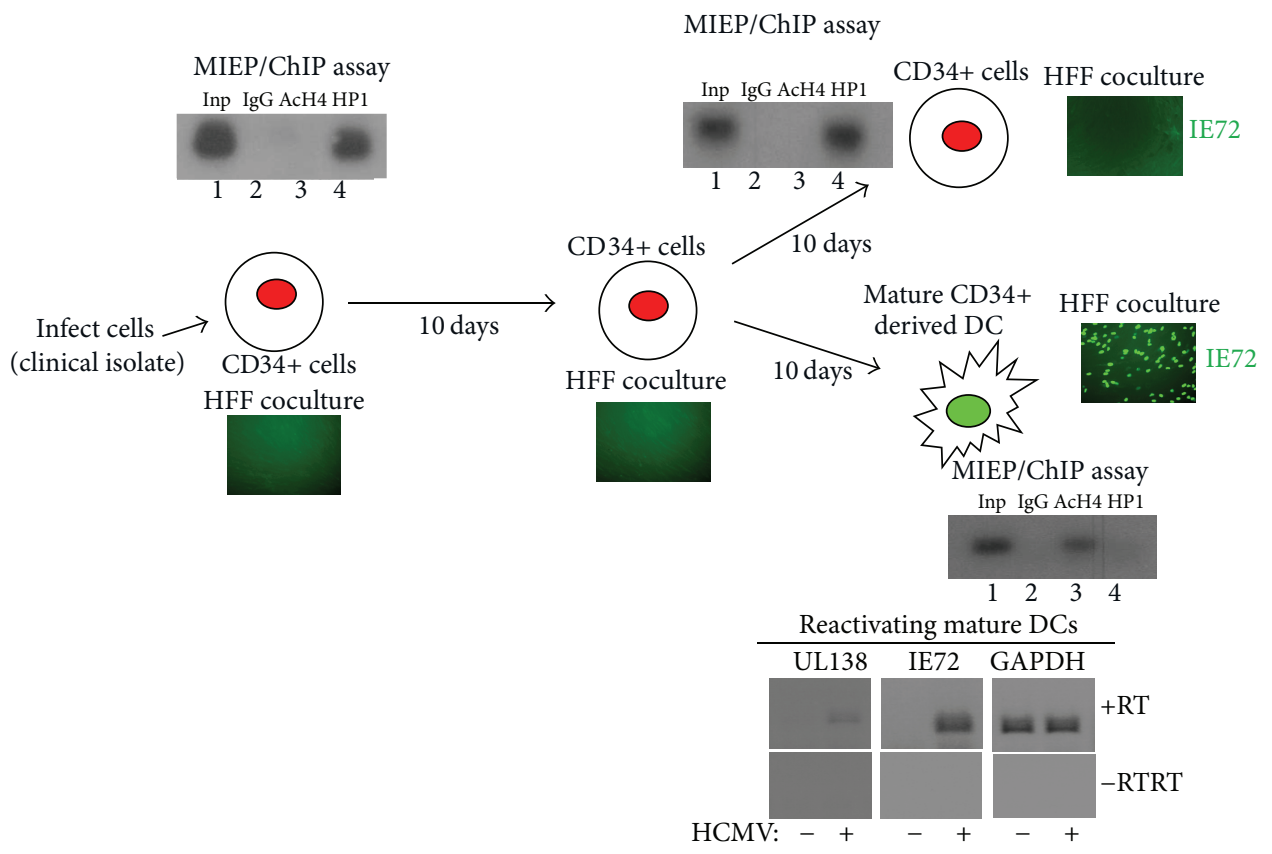

FIGURE 4: Establishment of experimental latency in primary progenitor CD34+ cells and reactivation in the myeloid lineage. Following infection of CD34+ progenitor cells with the TB40E strain of HCMV, latency is established, demonstrated by the hallmark of chromatin repressor HP1 association with the MIEP in chromatin immunoprecipitation assays (ChIP): Inp = input, IgG = immunoglobulin control, AcH3 = acetylated histone $\mathrm{H} 3$ (marker of active chromatin), and HP1 = heterochromatin protein 1 (marker of repressed chromatin). Coculture of these cells with fibroblasts (HFFs) does not result in IE gene expression. Untreated cells remain in a state of latency shown here for 20 days (top). Alternatively, if after 10 days cells are differentiated and matured into DCs, ChIP analysis shows that the MIEP becomes associated with markers of active chromatin ( $\mathrm{AcH} 4$ ) and coculture with fibroblasts shows immediate early 72-protein (IE72) gene expression. Consistent with these observations, transcripts can be detected for the viral latent gene transcript UL138, the viral lytic gene transcript IE72, and the cellular gene transcript GAPDH following reactivation by RT-PCR.

early (IE) protein, is silenced during latency but its expression is reactivated following differentiation. Essentially, the same differentiation-dependent regulation of viral lytic gene expression is observed using experimentally latently infected CD14+ monocytes before and after their differentiation to interstitial-like DCs. Most importantly, these observations in experimentally latent myeloid cells fully recapitulate similar analyses in naturally latently infected monocytes and CD34+ progenitor cells [60]. More recently, established myelomonocytic cells lines have also been described which can mimic certain, but perhaps not all $[61,62]$, aspects of latency observed in experimentally latent primary myeloid cells. These include monocytic cells lines, such as THP-1 cells $[63,64]$, as well as CD34+ cell lines, such as Kasumi-3 cells $[61,65]$.

Regardless, it has become clear that such analyses of experimental latent infection benefit from the use of clinical isolates of HCMV containing full length viral genomes. In contrast, viruses which have been extensively passaged in fibroblasts (so-called laboratory-adapted strains) routinely lose a $15 \mathrm{~kb}$ region of the genome, termed $\mathrm{U}_{L b^{\prime}}$ [69]. This is known to encode at least two latency-associated gene products $[46,59]$ and so may be compromised with respect to at least some latency-associated functions.

Genomes of multiple HCMV clinical strains have now been cloned as infectious bacterial artificial chromosomes
(BACs) and this has led to the ability to generate viral mutants as well as fluorescently-tagged viruses which have proved to be extremely useful in the analysis of HCMV lytic infection and, more recently, latent infection. For a number of studies, including our own, the use of an HCMV BAC constitutively expressing GFP under the control of the SV40 promoter has been useful in detecting cells carrying latent virus [65]. Similarly, a recently described clinical isolate of HCMV in which the viral major immediate early 2 protein is fused to a GFP tag [70] has been used to detect reactivation after terminal differentiation of myeloid cells [62].

\section{The Use of Experimental Models of HCMV Latency to Understand Changes That Occur in the Cell}

During latent infection the viral MIEP is heavily suppressed by histone posttranslational modifications essentially preventing lytic infection. However, a number of analyses using ex vivo naturally latent, as well as in vitro experimentally latent, model systems have identified latency-associated transcription of a number of specific viral genes (Figure 5). Whilst the function of many of these viral genes during latency is far from clear, recent studies have posited potential roles for 
TABLE 1: Cellular miRNAs are regulated during HCMV latency. Following the establishment of HCMV latency in CD34+ progenitor cells, cellular miRNA analysis was carried out using the Invitrogen NCode system [45] which identified a small number of changes.

\begin{tabular}{lcc}
\hline MicroRNA & $\begin{array}{c}\text { Fold change during latency } \\
\text { compared to mock }\end{array}$ & $P$ value \\
\hline hsa-miR-let-7a & -2.5 & 0.005 \\
hsa-miR-let-7b & -3.7 & 0.0001 \\
hsa-miR-206 & -2 & 0.02 \\
hsa-miR-296 3p & -2.6 & 0.007 \\
hsa-miR-297 & -2.9 & 0.001 \\
hsa-miR-32 & -2 & 0.07 \\
hsa-miR-608 & -2.4 & 0.008 \\
hsa-miR-92a & -2.5 & 0.003 \\
\hline
\end{tabular}

some of these viral gene products during latent infection [49, 71-73].

Whilst our own work using established experimental latency protocols has contributed to such studies on latencyassociated viral gene expression $[49,71,72]$, we have also analysed a number of changes in cellular gene expression during latent infection $[45,78]$. These have included changes in total cellular microRNAs associated with latency as well as changes in secreted cellular proteins resulting from latent infection (the latency-associated secretome). All these, we believe, are likely to have importance for efficient latent carriage and/or reactivation.

\subsection{Latency-Associated Changes in Cellular miRNAs Can} Affect the Cellular Transcriptome. MicroRNAs (miRNAs) are now accepted to be important posttranscriptional regulators of cellular gene expression which act in concert to fine tune expression of target genes. As many miRNAs can have multiple targets, they also represent an expedient and efficient way of coordinately regulating gene expression. For this reason, we analysed the total cellular miRNA profile of experimentally latent primary $\mathrm{CD} 34+$ cells and identified a number of changes accompanying latency ([45] and see Table 1).

One of the identified miRNAs which was dysregulated during HCMV latency using an experimental latency system with primary CD34+ cells was hsa-miR-92a (Table 1) which was routinely downregulated during latent infection. Interestingly, this latency-associated downregulation of hsa-miR92a resulted in the upregulation of cellular GATA2, a known target of hsa-miR-92a (Figure 6). GATA2 is a cellular transcription factor important for the regulation of hematopoiesis in the myeloid lineage and is argued to be important as a master switch involved in helping drive myelopoiesis $[74-76,79,80]$. Since HCMV establishes latency in the myeloid lineage, it has been hypothesised that this increase in GATA2 expression during latency may aid latent carriage $[45,46]$.

Not only is it likely that the latency-associated increase in GATA2 impinges on cellular gene expression associated with myelopoiesis but, as there are a number of predicted GATA2 binding sites in the promoters of predicted latencyassociated transcripts such as LUNA, it is also involved in the concomitant regulation of viral latent gene expression $[46,49]$. Interestingly, in contrast to the LUNA promoter, GATA2 binding sites in the promoter of another latencyassociated transcript, UL144, are only present in some clinical isolates of virus. In these isolates which do not carry GATA2 binding sites in the UL144 promoter, UL144 is not detectable during experimental latent infection ([46] and Figure 6). This suggests that, at least for some viral isolates, specific latency-associated gene expression may be isolate specific [46]. Besides the known role of GATA2 in myelopoiesis, GATA2 can also drive the expression of other cellular genes, including cellular IL-10 (cIL-10) (Figure 6), which is discussed further in the next section.

\subsection{Changes in the Cellular Secretome in Response to HCMV} Latency. As already discussed above, changes in a small number of cellular miRNAs could have profound effects on the expression of multiple cellular proteins. Studies on the latency-associated secretome from experimental latency using primary CD34+ cells have identified significant increases in a number of cellular proteins secreted during latent infection [78]. Although the mechanism by which many of these are upregulated has yet to be determined, one cytokine which has been analysed in some detail is cIL-10 and, as shown in Table 1 and Figure 6, its upregulation is mediated by hsa-miR-92a via GATA2 [45].

Our recent work has shown that this increased secretion of cIL-10 during latency has two discernible effects: (i) it leads to prolife signalling [45] and (ii) it aids suppression of CD4+ T cell effector functions [78] in latently infected CD34+ cells. It is becoming clear that latent infection is a far more active process than first believed and that latencyassociated viral gene expression leaves a distinct signature on the latently infected cell, at least in experimental models of latency in primary myeloid cells. Consequently, the established argument that latent infection is essentially invisible to the adaptive arm of the host immune response is likely to be far from true. Indeed, our work and others have shown that a number of latency-associated viral proteins are targets for the host cytotoxic T cell (CTL) response [72, 81]. However, in our hands, the $\mathrm{T}$ cells that are specific for these viral antigens appear to be cIL-10 and TGF-beta secreting cells-essentially of T suppressor phenotype [72]. The observation that latently infected cells also secrete high levels of both cIL-10 and TGFbeta suggests that the latent microenvironment is heavily immunosuppressive due to the latency-associated secretome [78]. Besides an effect on inhibition of CD4+ T cell effector function, this latency-associated increase in cIL-10 also has profound prolife effects on CD34+ cells by preventing FASmediated killing [45]. 


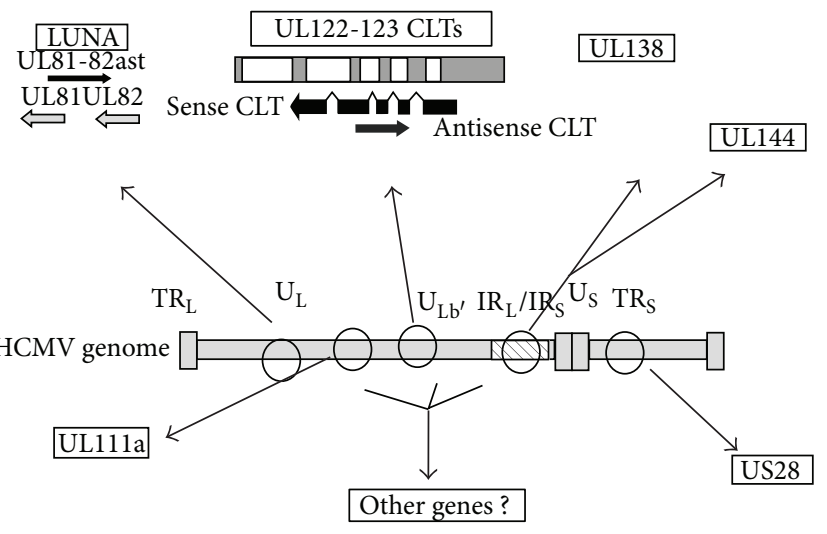

FIgURE 5: HCMV genes expressed during latency. The HCMV genome is comprised of two terminal repeats (TR), two internal repeats (IR), a unique long region (UL), and a unique short region (US). Genes expressed during latency identified so far are boxed and include US28 [66], UL82as/LUNA [67], latency-associated viral IL10/UL111a [68], and UL122/123 CMV latency transcripts (CTLs) [53] regions of the genome. The region of the genome termed $U_{L b^{\prime}}$ is a segment of UL which becomes lost during extensive passaging of laboratory strains and two genes identified during latency are located in $\mathrm{U}_{L b^{\prime}}$ UL144 [46] and UL138 [59].

\section{Understanding Latency-Associated Changes Could Allow the Development of Novel Strategies to Target HCMV Latency in the Clinical Setting}

\subsection{Targeting of the HCMV Latently Expressed Protein UL138.} Our increased understanding of the role of viral gene products during latency, from analyses in experimental and ex vivo latency model systems, has now led to the possibility that these may be the basis for novel chemo- or immunotherapeutic targets with a view to clearing latency [71] with the important caveat that the principles established in latency models are transferrable to the in vivo setting.

Similarly, such interventions would be made all the more powerful when performed in conjunction with biomarkers for latent infection. At present, such biomarkers specifically for latency are limited but could include quantitation of latent load in circulating monocytes by quantitative PCR or, perhaps, frequency of circulating HCMV-specific T cells in the peripheral blood compartment on the basis that high $\mathrm{T}$ cell frequencies to murine cytomegalovirus are maintained by constant restimulation with reactivating virus [82].

An understanding of how one latency-associated viral gene, UL138, impacts cellular gene expression has recently led to the ability to target and kill latently infected cells in both experimental and natural latency. In these studies the effects of expression of UL138 on total cellular plasma-membrane associated proteins in monocytic cells were comprehensively analysed by stable isotope labelling with amino acids in cell culture (SILAC) [71]. Amongst the most robust changes in cellular protein expression resulting from UL138 expression was the downregulation of the multidrug resistance protein 1 (MRP-1) [71]. Aside from its function as a drug transporter,
MRP-1 is also associated with the regulation of myeloid differentiation and dendritic cells (DCs) $[83,84]$. Although a role for the downregulation of MRP-1 during HCMV latent infection is far from clear, the drug transporter function of this cellular protein makes it a reasonable target for intervention in latency with potential for experimental investigation using specific drugs [71]. In myeloid cells, MRP-1 can function to transport toxic drugs, such as the vinca alkaloid vincristine, out of the cell. On this basis, the downregulation of MRP-1 in latently infected CD34+ cells should make these cells more prone to killing with vincristine. Consistent with this, treatment of both experimentally latently infected CD34+ cells and naturally latent monocytes from a cohort of seropositive donors with vincristine led to the specific killing of latently infected cells. As expected, this also led to a profound reduction in reactivation of virus from these drugtreated cells [71].

Clearly, these preliminary results will clearly need verification in the clinical setting. Similarly, vincristine is a toxic drug and might only be considered as an experimental treatment of last resort. However, if less toxic derivatives of vincristine could be developed which also cleared latently infected cells experimentally, it may be that a seropositive recipient could be prophylactically treated to clear or at least reduce their latent HCMV load, prior to any immunosuppression.

Regardless, these data clearly show a robust proof of principle that latent HCMV infection of myeloid cells results in phenotypic changes in the cell which may make them therapeutically targetable and opens up the possibility that such strategies could be developed either to clear latently infected cells from CD34+ cells prior to engraftment or to reduce the latent load of transplant recipients pretransplant to diminish levels of host virus reactivation upon immune suppression.

\section{Avenues for Future Investigation}

The long-term aims of our laboratory have been to try to gain a full understanding of host/pathogen interactions during HCMV latent infection and these studies have helped to identify a number of major changes in cell gene expression resulting from latent carriage. Such studies have allowed us to start developing novel therapeutic strategies to target these latency-associated changes in cell phenotype with a serious view to clearing latent infection, at least in some clinical settings. It would clearly be difficult to advocate such treatment in the healthy immune competent individual and with other herpesviruses such as EBV it is arguable whether this could be achieved [85] although multiple strategies have been posited to target latent viral functions in EBV-associated malignancies [86]. However, with HCMV, these interventions may be opportune in the context of transplant-mediated reactivation where sufficient reduction in latent load of the virus in the graft, or in the recipient prior to transplant, could reduce levels of reactivation sufficiently to reduce disease in such an immune-suppressed setting. 


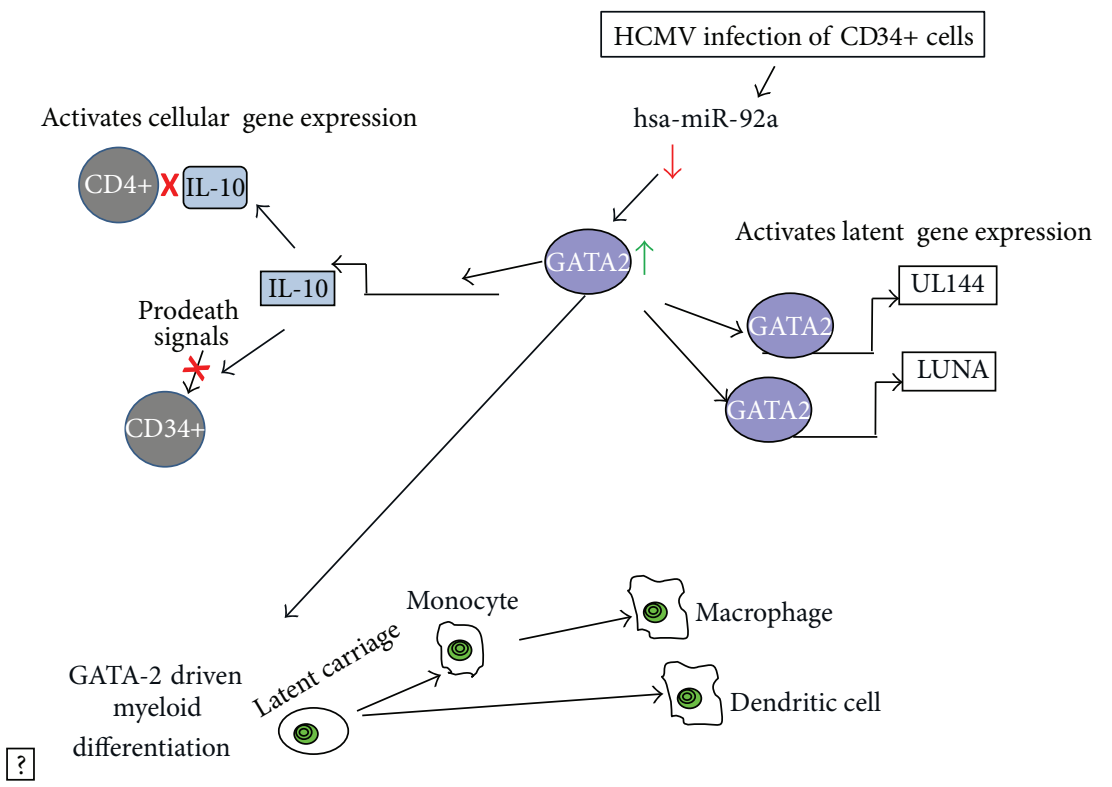

FIgURE 6: GATA2 may be a key regulator of HCMV latency Downregulation of the human miRNA hsa-miR-92a leads to a concomitant upregulation of the myeloid transcription factor GATA2 [45]. GATA2 is important during myeloid differentiation [74-77] and can drive the expression of latency-associated genes UL144 and LUNA [46, 49]. GATA2 can also drive the expression of cellular IL-10 which in turn can energize CD4+ cytotoxic T cells and act as a prolife signal for CD34+ cells [78].

As our understanding of the complexities in host-cell interactions during HCMV latency expands, an increasing number of latency-associated changes in the cell are likely to be identified which may also be targetable with novel immuno- and chemotherapeutic strategies. This may include viral targets or targets resulting from modification of cellular gene expression and we believe that this will allow the possibility of targeting and clearing latent infection, at least in some settings, which is a concept for HCMV which would not have been thought possible a decade ago.

\section{Conflict of Interests}

The authors declare that there is no conflict of interests regarding the publication of this paper.

\section{Acknowledgments}

The authors would like to thank members, past and present, of their laboratory and the numerous colleagues in the field whose work has contributed to this review. The authors also apologize to those colleagues whose work has not been cited due to space limitations. Finally, the authors gratefully acknowledge funding from the UK Medical Research Council (J.H.S. G: 0701279; J.H.S. and M.R.W. MR/K021087/1) which supports the current research in their laboratories and also the support of NIHR UK Biomedical Research Centre.

\section{References}

[1] J. A. Zaia, "The biology of human cytomegalovirus infection after bone marrow transplantation," International Journal of Cell Cloning, vol. 4, supplement 1, pp. 135-154, 1986.
[2] R. H. Rubin, "Impact of cytomegalovirus infection on organ transplant recipients," Reviews of Infectious Diseases, vol. 12, supplement 7, pp. S754-S766, 1990.

[3] S. L. Orloff, Y.-K. Hwee, C. Kreklywich et al., "Cytomegalovirus latency promotes cardiac lymphoid neogenesis and accelerated allograft rejection in CMV Naïve recipients," The American Journal of Transplantation, vol. 11, no. 1, pp. 45-55, 2011.

[4] M. Comar, S. Delbue, L. Lepore et al., "Latent viral infections in young patients with inflammatory diseases treated with biological agents: prevalence of JC virus genotype 2," Journal of Medical Virology, vol. 85, no. 4, pp. 716-722, 2013.

[5] Z. J. Hagay, G. Biran, A. Ornoy, and E. A. Reece, "Congenital cytomegalovirus infection: a long-standing problem still seeking a solution," The American Journal of Obstetrics and Gynecology, vol. 174, part 1, pp. 241-245, 1996.

[6] J. A. Zaia and D. J. Lang, "Cytomegalovirus infection of the fetus and neonate," Neurologic Clinics, vol. 2, no. 2, pp. 387-410, 1984.

[7] C. A. Alford, S. Stagno, and R. F. Pass, "Natural history of perinatal cytomegaloviral infection," Ciba Foundation Symposium, no. 77, pp. 125-147, 1979.

[8] D. L. Levy, "Persistent fetal tachycardia in utero prior to labor in an infant with congenital cytomegalic inclusion disease: case report," The American Journal of Obstetrics and Gynecology, vol. 112 , no. 6, pp. 859-860, 1972.

[9] W. Britt, "Manifestations of human cytomegalovirus infection: proposed mechanisms of acute and chronic disease," Current Topics in Microbiology and Immunology, vol. 325, pp. 417-470, 2008.

[10] J. G. P. Sissons, J. H. Sinclair, and L. K. Borysiewicz, "Pathogenesis of human cytomegalovirus disease and the kidney," Kidney International, vol. 35, pp. S8-S12, 1991.

[11] D. J. Stratton and R. S. Lawrence, Vaccines for the 21st Century: A Tool for Decision Making, National Academies Press, Washington, DC, USA, 2000. 
[12] A. H. Rook, "Interactions of cytomegalovirus with the human immune system," Reviews of Infectious Diseases, vol. 10, supplement 3, pp. S460-467, 1988.

[13] C. Grahame-Clarke, "Human cytomegalovirus, endothelial function and atherosclerosis," Herpes, vol. 12, no. 2, pp. 42-45, 2005.

[14] D. N. Streblow, S. L. Orloff, and J. A. Nelson, "Acceleration of allograft failure by cytomegalovirus," Current Opinion in Immunology, vol. 19, no. 5, pp. 577-582, 2007.

[15] J. A. Fishman, "Infections in immunocompromised hosts and organ transplant recipients: essentials," Liver Transplantation, vol. 17, supplement 3, pp. S34-S37, 2011.

[16] R. F. Fonseca, M. T. Kawamura, J. A. Oliveira, A. Teixeira, G. Alves, and M. D. G. D. C. Carvalho, "The prevalence of human cytomegalovirus DNA in gliomas of Brazilian patients," Memorias do Instituto Oswaldo Cruz, vol. 107, no. 7, pp. 953-954, 2012.

[17] B. Bhattacharjee, N. Renzette, and T. F. Kowalik, "Genetic analysis of cytomegalovirus in malignant gliomas," Journal of Virology, vol. 86, no. 12, pp. 6815-6824, 2012.

[18] Q. Lepiller, M. K. Tripathy, V. di Martino, B. Kantelip, and G. Herbein, "Increased HCMV seroprevalence in patients with hepatocellular carcinoma," Virology Journal, vol. 8, article 485, 2011.

[19] C. Hawkins and S. Croul, "Viruses and human brain tumors: cytomegalovirus enters the fray," Journal of Clinical Investigation, vol. 121, no. 10, pp. 3831-3833, 2011.

[20] L. Soroceanu and C. S. Cobbs, "Is HCMV a tumor promoter?" Virus Research, vol. 157, no. 2, pp. 193-203, 2011.

[21] K. Barami, "Oncomodulatory mechanisms of human cytomegalovirus in gliomas," Journal of Clinical Neuroscience, vol. 17, no. 7, pp. 819-823, 2010.

[22] C. Söderberg-Nauclér, "HCMV microinfections in inflammatory diseases and cancer," Journal of Clinical Virology, vol. 41, no. 3, pp. 218-223, 2008.

[23] W. L. Drew, "Cytomegalovirus infection in patients with AIDS," Journal of Infectious Diseases, vol. 158, no. 2, pp. 449-456, 1988.

[24] L. Strasfeld and S. Chou, "Antiviral drug resistance: mechanisms and clinical implications," Infectious Disease Clinics of North America, vol. 24, no. 3, pp. 809-833, 2010.

[25] P. Rutgeerts, K. Geboes, E. Ponette, G. Coremans, and G. Vantrappen, "Acute infective colitis caused by endemic pathogens in Western Europe: endoscopic features," Endoscopy, vol. 14, no. 6, pp. 212-219, 1982.

[26] D. N. Streblow, S. L. Orloff, and J. A. Nelson, "Do pathogens accelerate atherosclerosis?” Journal of Nutrition, vol. 131, no. 10, pp. 2798S-2804S, 2001.

[27] A. V. Imbronito, S. L. Marcelino, S. R. Grande, F. D. Nunes, and A. G. Romito, "Detection of human cytomegalovirus and epstein-barr virus in coronary atherosclerotic tissue," Brazilian Journal of Microbiology, vol. 41, no. 3, pp. 563-566, 2010.

[28] M. Popović, K. Smiljanić, B. Dobutović, T. Syrovets, T. Simmet, and E. R. Isenović, "Human cytomegalovirus infection and atherothrombosis," Journal of Thrombosis and Thrombolysis, vol. 33, no. 2, pp. 160-172, 2012.

[29] L. Scrivano, C. Sinzger, H. Nitschko, U. H. Koszinowski, and B. Adler, "HCMV spread and cell tropism are determined by distinct virus populations," PLoS Pathogens, vol. 7, no. 1, Article ID e1001256, 2011.
[30] M. D. Tolpin, J. A. Stewart, D. Warren et al., “Transfusion transmission of cytomegalovirus confirmed by restriction endonuclease analysis," The Journal of Pediatrics, vol. 107, no. 6, pp. 953956, 1985.

[31] P. D. Griffiths, A. Stanton, E. McCarrell et al., "Cytomegalovirus glycoprotein-B vaccine with MF59 adjuvant in transplant recipients: a phase 2 randomised placebo-controlled trial," The Lancet, vol. 377, no. 9773, pp. 1256-1263, 2011.

[32] M. A. Kharfan-Dabaja, M. Boeckh, M. B. Wilck et al., "A novel therapeutic cytomegalovirus DNA vaccine in allogeneic haemopoietic stem-cell transplantation: a randomised, doubleblind, placebo-controlled, phase 2 trial," The Lancet Infectious Diseases, vol. 12, no. 4, pp. 290-299, 2012.

[33] S. A. Plotkin, M. L. Smiley, H. M. Friedman et al., “Townevaccine-induced prevention of cytomegalovirus disease after renal transplants," The Lancet, vol. 1, no. 8376, pp. 528-530, 1984.

[34] M. Bain, M. Mendelson, and J. Sinclair, "Ets-2 Respressor Factor (ERF) mediates repression of the human cytomegalovirus major immediate-early promoter in undifferentiated nonpermissive cells," Journal of General Virology, vol. 84, part 1, pp. 41-49, 2003.

[35] E. Wright, M. Bain, L. Teague, J. Murphy, and J. Sinclair, "Ets-2 repressor factor recruits histone deacetylase to silence human cytomegalovirus immediate-early gene expression in non-permissive cells," Journal of General Virology, vol. 86, part 3, pp. 535-544, 2005.

[36] M. B. Reeves, P. A. MacAry, P. J. Lehner, J. G. P. Sissons, and J. H. Sinclair, "Latency, chromatin remodeling, and reactivation of human cytomegalovirus in the dendritic cells of healthy carriers," Proceedings of the National Academy of Sciences of the United States of America, vol. 102, no. 11, pp. 4140-4145, 2005.

[37] M. B. Reeves, P. J. Lehner, J. G. P. Sissons, and J. H. Sinclair, "An in vitro model for the regulation of human cytomegalovirus latency and reactivation in dendritic cells by chromatin remodelling," Journal of General Virology, vol. 86, part 11, pp. 29492954, 2005.

[38] P. Ghazal, H. Lubon, C. Reynolds-Kohler, L. Hennighausen, and J. A. Nelson, "Interactions between cellular regulatory proteins and a unique sequence region in the human cytomegalovirus major immediate-early promoter," Virology, vol. 174, no. 1, pp. 18-25, 1990.

[39] R. T. Saffert, R. R. Penkert, and R. F. Kalejta, "Cellular and viral control over the initial events of human cytomegalovirus experimental latency in CD34+ cells," Journal of Virology, vol. 84, no. 11, pp. 5594-5604, 2010.

[40] C. C. Rossetto, M. Tarrant-Elorza, and G. S. Pari, "Cis and Trans Acting Factors Involved in Human Cytomegalovirus Experimental and Natural Latent Infection of CD14 (+) Monocytes and CD34 (+) Cells," PLoS Pathogens, vol. 9, no. 5, Article ID e1003366, 2013.

[41] C. Söderberg-Nauclér, K. N. Fish, and J. A. Nelson, "Reactivation of latent human cytomegalovirus by allogeneic stimulation of blood cells from healthy donors," Cell, vol. 91, no. 1, pp. 119126, 1997.

[42] C. Sinzger, M. Digel, and G. Jahn, "Cytomegalovirus cell tropism," Current Topics in Microbiology and Immunology, vol. 325, pp. 63-83, 2008.

[43] K. Kondo, H. Kaneshima, and E. S. Mocarski, "Human cytomegalovirus latent infection of granulocyte-macrophage progenitors," Proceedings of the National Academy of Sciences of the United States of America, vol. 91, no. 25, pp. 11879-11883, 1994. 
[44] G. Hahn, R. Jores, and E. S. Mocarski, "Cytomegalovirus remains latent in a common precursor of dendritic and myeloid cells," Proceedings of the National Academy of Sciences of the United States of America, vol. 95, no. 7, pp. 3937-3942, 1998.

[45] E. Poole, S. R. M. Dallas, J. Colston, R. S. V. Joseph, and J. Sinclair, "Virally induced changes in cellular microRNAs maintain latency of human cytomegalovirus in CD34+ progenitors," Journal of General Virology, vol. 92, part 7, pp. 1539-1549, 2011.

[46] E. Poole, A. Walther, K. Raven, C. A. Benedict, G. M. Mason, and J. Sinclair, "The myeloid transcription factor GATA-2 regulates the viral UL144 gene during human cytomegalovirus latency in an isolate-specific manner," Journal of Virology, vol. 87, no. 8, pp. 4261-4271, 2013.

[47] M. Mendelson, S. Monard, P. Sissons, and J. Sinclair, "Detection of endogenous human cytomegalovirus in CD34+ bone marrow progenitors," Journal of General Virology, vol. 77, part 12, pp. 3099-3102, 1996.

[48] J. Taylor-Wiedeman, J. G. P. Sissons, L. K. Borysiewicz, and J. H. Sinclair, "Monocytes are a major site of persistence of human cytomegalovirus in peripheral blood mononuclear cells," Journal of General Virology, vol. 72, part 9, pp. 2059-2064, 1991.

[49] M. B. Reeves and J. H. Sinclair, "Analysis of latent viral gene expression in natural and experimental latency models of human cytomegalovirus and its correlation with histone modifications at a latent promoter," Journal of General Virology, vol. 91, part 3, pp. 599-604, 2010.

[50] J. A. Nelson, J. W. Gnann Jr., and P. Ghazal, "Regulation and tissue-specific expression of human cytomegalovirus," Current Topics in Microbiology and Immunology, vol. 154, pp. 75-100, 1990.

[51] N. Quirici, D. Soligo, L. Caneva, F. Servida, P. Bossolasco, and G. L. Deliliers, "Differentiation and expansion of endothelial cells from human bone marrow CD133+ cells," British Journal of Haematology, vol. 115, no. 1, pp. 186-194, 2001.

[52] M. B. Reeves, H. Coleman, J. Chadderton, M. Goddard, J. G. P. Sissons, and J. H. Sinclair, "Vascular endothelial and smooth muscle cells are unlikely to be major sites of latency of human cytomegalovirus in vivo," Journal of General Virology, vol. 85, part 11, pp. 3337-3341, 2004.

[53] B. Slobedman and E. S. Mocarski, "Quantitative analysis of latent human cytomegalovirus," Journal of Virology, vol. 73, no. 6, pp. 4806-4812, 1999.

[54] S. Larsson, C. Söderberg-Nauclér, F.-Z. Wang, and E. Möller, "Cytomegalovirus DNA can be detected in peripheral blood mononuclear cells from all seropositive and most seronegative healthy blood donors over time," Transfusion, vol. 38, no. 3, pp. 271-278, 1998.

[55] A. K. L. Cheung, A. Abendroth, A. L. Cunningham, and B. Slobedman, "Viral gene expression during the establishment of human cytomegalovirus latent infection in myeloid progenitor cells," Blood, vol. 108, no. 12, pp. 3691-3699, 2006.

[56] J. L. Stern and B. Slobedman, "Human cytomegalovirus latent infection of myeloid cells directs monocyte migration by Up-regulating monocyte chemotactic protein-1," Journal of Immunology, vol. 180, no. 10, pp. 6577-6585, 2008.

[57] D. Hargett and T. E. Shenk, "Experimental human cytomegalovirus latency in CD14+ monocytes," Proceedings of the National Academy of Sciences of the United States of America, vol. 107, no. 46, pp. 20039-20044, 2010.

[58] F. D. Goodrum, C. T. Jordan, K. High, and T. Shenk, "Human cytomegalovirus gene expression during infection of primary hematopoietic progenitor cells: a model for latency," Proceedings of the National Academy of Sciences of the United States of America, vol. 99, no. 25, pp. 16255-16260, 2002.

[59] F. Goodrum, M. Reeves, J. Sinclair, K. High, and T. Shenk, "Human cytomegalovirus sequences expressed in latently infected individuals promote a latent infection in vitro," Blood, vol. 110, no. 3, pp. 937-945, 2007.

[60] M. B. Reeves and T. Compton, "Inhibition of inflammatory interleukin-6 activity via extracellular signal-regulated kinasemitogen-activated protein kinase signaling antagonizes human cytomegalovirus reactivation from dendritic cells," Journal of Virology, vol. 85, no. 23, pp. 12750-12758, 2011.

[61] E. R. Albright and R. F. Kalejta, "Myeloblastic cell lines mimic some but not all aspects of human cytomegalovirus experimental latency defined in primary CD34+ cell populations," Journal of Virology, vol. 87, no. 17, pp. 9802-9812, 2013.

[62] R. R. Penkert and R. F. Kalejta, "Human embryonic stem cell lines model experimental human cytomegalovirus latency," mBio, vol. 4, no. 3, 2013.

[63] J. H. Sinclair, J. Baillie, L. A. Bryant, J. A. Taylor-Wiedeman, and J. G. P. Sissons, "Repression of human cytomegalovirus major immediate early gene expression in a monocytic cell line," Journal of General Virology, vol. 73, part 2, pp. 433-435, 1992.

[64] C. G. Abraham and C. A. Kulesza, "Polycomb repressive complex 2 silences human cytomegalovirus transcription in quiescent infection models," Journal of Virology, vol. 87, no. 24, pp. 13193-13205, 2013.

[65] C. M. O'Connor and E. A. Murphy, "A myeloid progenitor cell line capable of supporting human cytomegalovirus latency and reactivation, resulting in infectious progeny," Journal of Virology, vol. 86, no. 18, pp. 9854-9865, 2012.

[66] P. S. Beisser, L. Laurent, J.-L. Virelizier, and S. Michelson, "Human cytomegalovirus chemokine receptor gene US28 is transcribed in latently infected THP-1 monocytes," Journal of Virology, vol. 75, no. 13, pp. 5949-5957, 2001.

[67] M. G. Bego and S. St. Jeor, "Human cytomegalovirus infection of cells of hematopoietic origin: HCMV-induced immunosuppression, immune evasion, and latency," Experimental Hematology, vol. 34, no. 5, pp. 555-570, 2006.

[68] C. Jenkins, A. Abendroth, and B. Slobedman, "A novel viral transcript with homology to human Interleukin-10 Is expressed during latent human cytomegalovirus infection," Journal of Virology, vol. 78, no. 3, pp. 1440-1447, 2004.

[69] T.-A. N. Cha, E. Tom, G. W. Kemble, G. M. Duke, E. S. Mocarski, and R. R. Spaete, "Human cytomegalovirus clinical isolates carry at least 19 genes not found in laboratory strains," Journal of Virology, vol. 70, no. 1, pp. 78-83, 1996.

[70] S. Straschewski, M. Warmer, G. Frascaroli, H. Hohenberg, T. Mertens, and M. Winkler, "Human cytomegaloviruses expressing yellow fluorescent fusion proteins-characterization and use in antiviral screening," PLOS ONE, vol. 5, no. 2, Article ID e0009174, 2010.

[71] M. P. Weekes, S. Y. L. Tan, E. Poole et al., "Latency-associated degradation of the MRP1 drug transporter during latent human cytomegalovirus infection," Science, vol. 340, no. 6129, pp. 199202, 2013.

[72] G. M. Mason, S. Jackson, G. Okecha et al., "Human cytomegalovirus latency-associated proteins elicit immunesuppressive IL-10 producing CD4(+) T cells," PLoS Pathogens, vol. 9, no. 10, Article ID e1003635, 2013.

[73] S. Avdic, J. Z. Cao, A. K. L. Cheung, A. Abendroth, and B. Slobedman, "Viral interleukin-10 expressed by human 
cytomegalovirus during the latent phase of infection modulates latently infected myeloid cell differentiation," Journal of Virology, vol. 85, no. 14, pp. 7465-7471, 2011.

[74] F.-Y. Tsai and S. H. Orkin, "Transcription factor GATA-2 is required for proliferation/survival of early hematopoietic cells and mast cell formation, but not for erythroid and myeloid terminal differentiation," Blood, vol. 89, no. 10, pp. 3636-3643, 1997.

[75] X. Pan, N. Minegishi, H. Harigae et al., "Identification of human GATA-2 gene distal IS exon and its expression in hematopoietic stem cell fractions," Journal of Biochemistry, vol. 127, no. 1, pp. 105-112, 2000.

[76] E. de Pater, P. Kaimakis, C. S. Vink et al., "GATA-2 is required for HSC generation and survival," The Journal of Experimental Medicine, vol. 210, no. 13, pp. 2843-2850, 2013.

[77] F.-Y. Tsal, G. Keller, F. C. Kuo et al., "An early haematopoietic defect in mice lacking the transcription factor GATA-2," Nature, vol. 371, no. 6494, pp. 221-226, 1994.

[78] G. M. Mason, E. Poole, J. G. P. Sissons, M. R. Wills, and J. H. Sinclair, "Human cytomegalovirus latency alters the cellular secretome, inducing cluster of differentiation (CD)4+ T-cell migration and suppression of effector function," Proceedings of the National Academy of Sciences of the United States of America, vol. 109, no. 36, pp. 14538-14543, 2012.

[79] N. K. Wilson, S. D. Foster, X. Wang et al., "Combinatorial transcriptional control in blood stem/progenitor cells: genomewide analysis of ten major transcriptional regulators," Cell Stem Cell, vol. 7, no. 4, pp. 532-544, 2010.

[80] C. Vicente, A. Conchillo, M. A. García-Sánchez, and M. D. Odero, "The role of the GATA-2 transcription factor in normal and malignant hematopoiesis," Critical Reviews in Oncology/Hematology, vol. 82, no. 1, pp. 1-17, 2012.

[81] S.-K. Tey, F. Goodrum, and R. Khanna, "CD8+ T-cell recognition of human cytomegalovirus latency-associated determinant pUL138," Journal of General Virology, vol. 91, part 8, pp. 20402048, 2010.

[82] M. Beswick, P. Annette, S. N. Lauder, C. Sweet, and P. A. Moss, "Antiviral therapy can reverse the development of immune senescence in elderly mice with latent cytomegalovirus infection," Journal of Virology, vol. 87, no. 2, pp. 779-789, 2013.

[83] O. Legrand, R. Zittoun, and J.-P. Marie, "Role of MRP1 in multidrug resistance in acute myeloid leukemia," Leukemia, vol. 13, no. 4, pp. 578-584, 1999.

[84] D. M. van der Kolk, E. G. E. de Vries, L. Noordhoek et al., "Activity and expression of the multidrug resistance proteins Pglycoprotein, MRP1, MRP2, MRP3 and MRP5 in de novo and relapsed acute myeloid leukemia," Leukemia, vol. 15, no. 10, pp. 1544-1553, 2001.

[85] J. B. Hawkins, E. Delgado-Eckert, D. A. Thorley-Lawson, and M. Shapiro, "The cycle of EBV infection explains persistence, the sizes of the infected cell populations and which come under CTL regulation," PLoS Pathogens, vol. 9, no. 10, Article ID e1003685, 2013.

[86] B. F. Israel and S. C. Kenney, "Virally targeted therapies for EBVassociated malignancies," Oncogene, vol. 22, no. 33, pp. 51225130, 2003. 

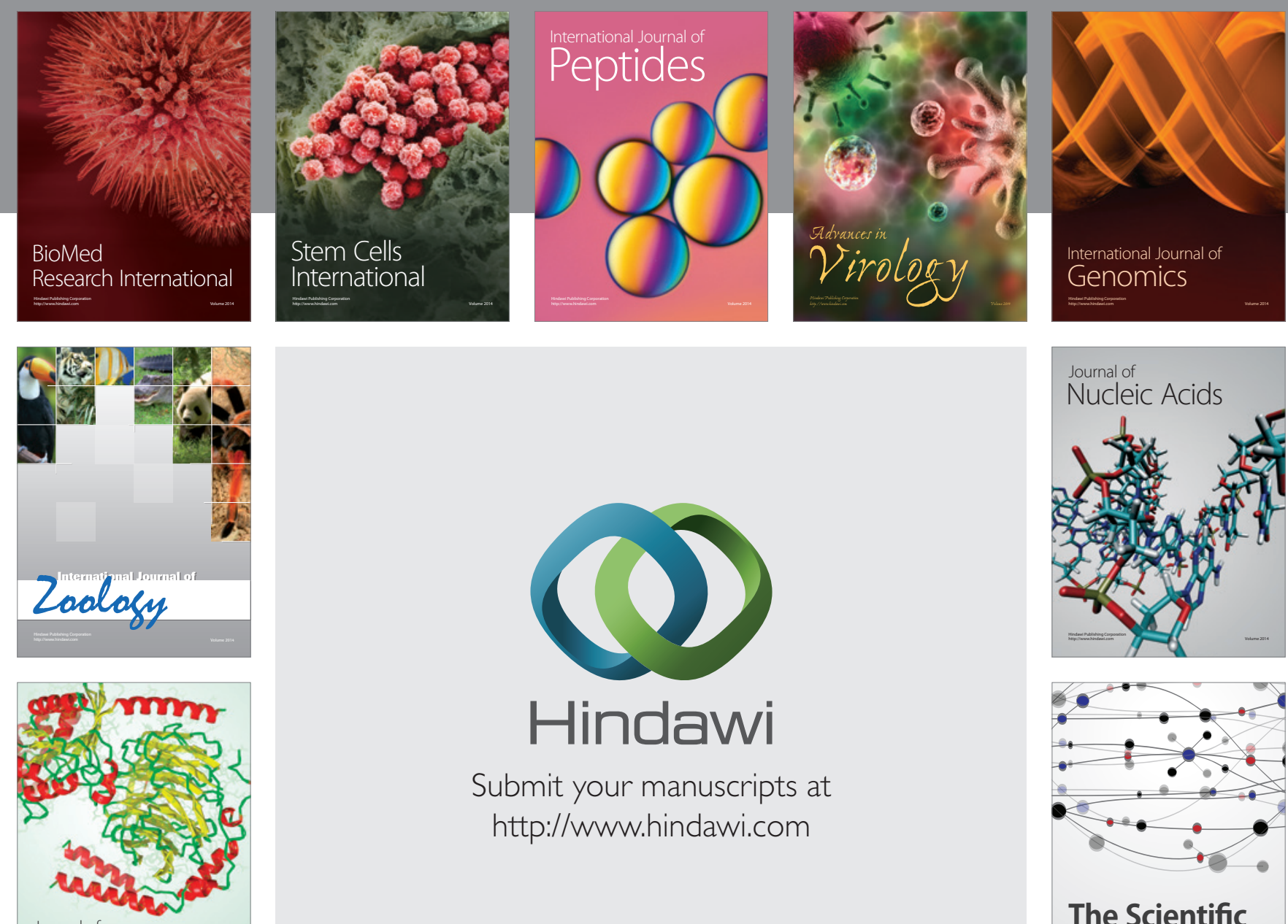

Submit your manuscripts at

http://www.hindawi.com

Journal of
Signal Transduction
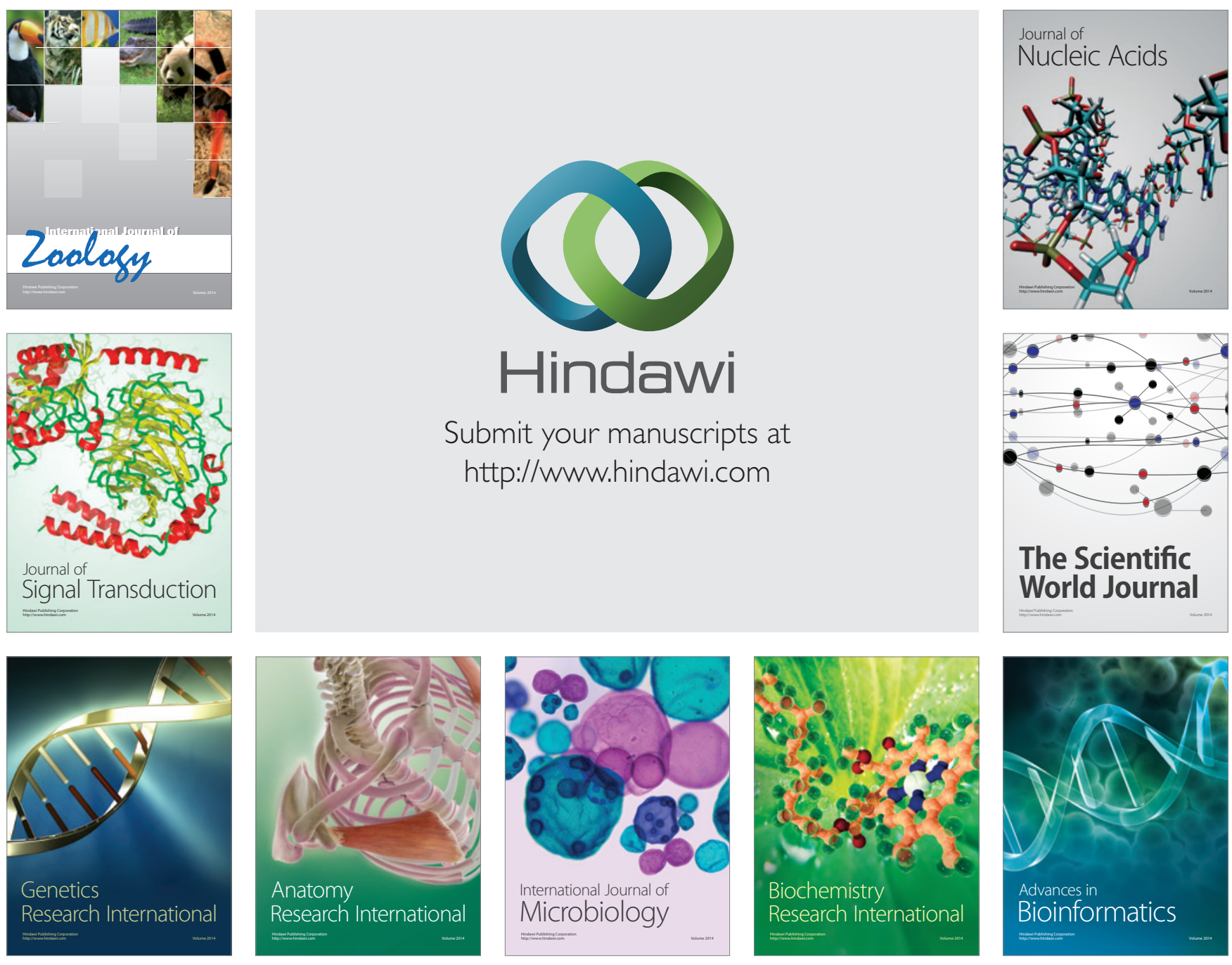

The Scientific World Journal
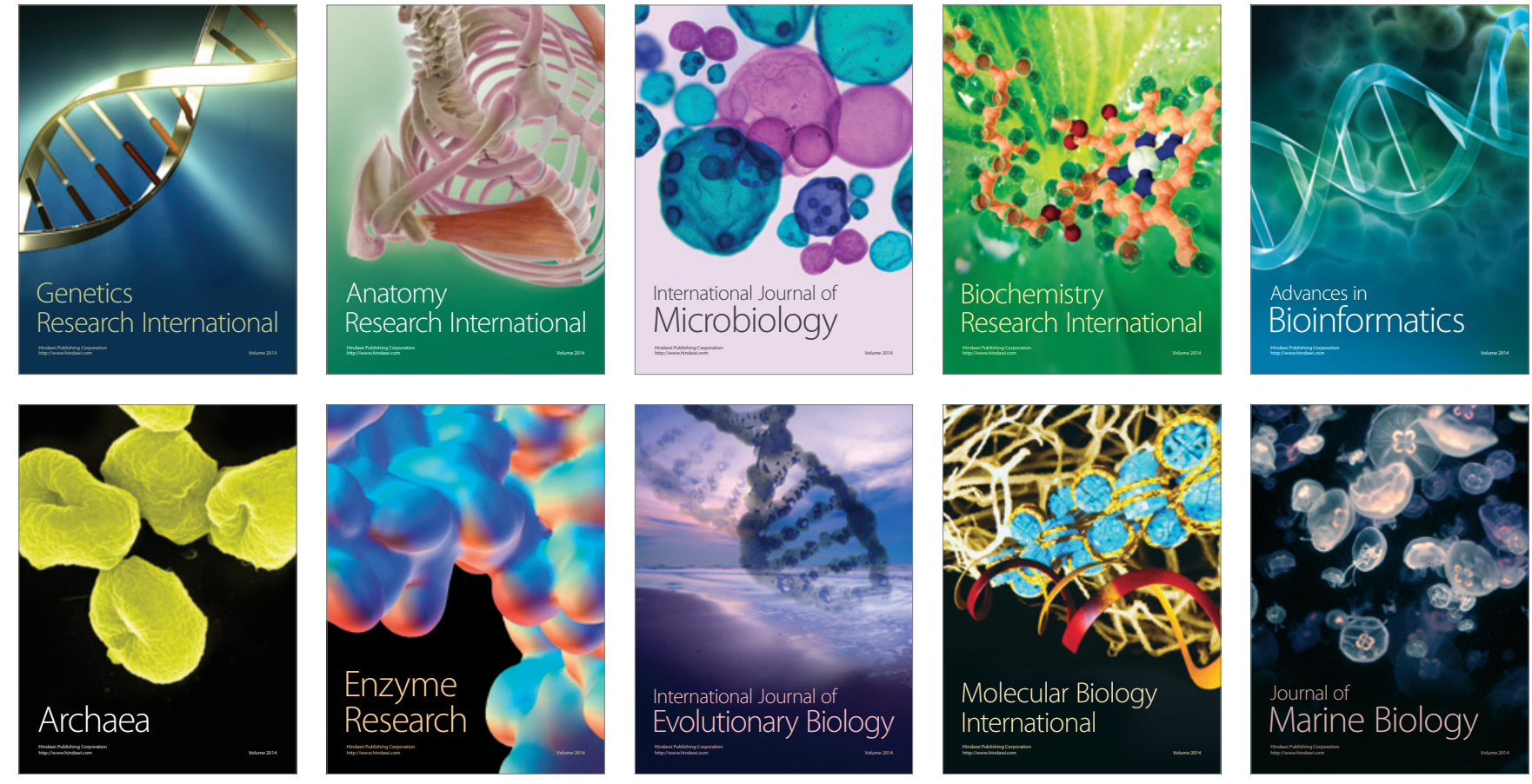